\title{
We Hommage au Professeur Roger Misès (1924-2012)
}

Claude Wacjman

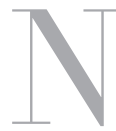

é le 13 avril 1924, le Professeur Roger Misès nous a quitté le 23 juillet 2012.

En 1957, il est nommé chef de service à la Fondation Vallée où il travaillera jusqu'à sa retraite, puis il y sera consultant et Professeur Emérite. Il réalise le travail en hôpital pédopsychiatrique avec une équipe constituée d'éducateurs spécialisés et d'infirmiers, appuyés par un plateau technique conséquent. Il conçoit les nouveaux bâtiments de son service et met en place la psychiatrie dans un des premiers secteurs infanto-juvénile. Il favorise le développement des Hôpitaux de jour et les traitements à temps partiel (CATTP).

N'oublions pas son passage à Montesson qui choisit de l'honorer en donnant de son vivant son nom à une unité de soins. C'est à lui qu'on doit l'importante modification des conditions d'hospitalisation des enfants en psychiatrie : de l'hospitalisation d'office de la loi de 1838 sur les aliénés, on passe à une hospitalisation ordinaire dans un cadre institutionnel.

En 1965 il devient membre de la SPP.

D'une très riche bibliographie on peut extraire trois ouvrages majeurs :

La cure en institution, ESF, 1980.

Les pathologies limites de l'enfance, PUF, 1999.

Classification des troubles mentaux de l'enfant et de l'adolescent, [1987], dont la 5 édition sous sa direction, paraît en 2012, très peu de temps après son décès. C'est la seule classification des troubles mentaux de l'enfance et de l'adolescence située dans une approche psychanalytique du développement. On note l'importance du concept de dysharmonie évolutive qu'elle contient. Elle est transposable dans la CIM-10 et sera bientôt transcodable informatiquement. Il faut aussi se rendre compte, quoi qu'en disent certaines associations de parents d'enfants autistes favorables à l'emploi du seul DSM-IV, que c'est cette classification qui est requise par les caisses d'assurance maladie pour assurer leurs prises en charge et par les MDPH lorsqu'elles rendent leurs autorisations ou statuent sur l'orientation et le droit à compensation des personnes handicapées. 
La première fois que j'ai rencontré le Pr Misès c'était sur les lieux mêmes de son travail, à la Fondation Vallée, durant l'année scolaire 1966-1967, lors de mes études d'éducateur spécialisé au Centre de Formation d'Educateurs Spécialisés d'Épinay sur Seine. Son directeur, le Dr Préaut, avait choisi de nous faire connaître cet endroit pour plusieurs raisons : la découverte du travail en pédopsychiatrie, relativement nouveau au sortir d'une période de garderie carcérale, la rencontre avec un chef de service psychanalyste pour faire la distinction entre maladie neurologique et maladie mentale, ouvrir à la classification des maladies mentales de l'enfant et à l'institutionnalisation des soins. Il nous apparut comme un personnage imposant, d'un sérieux d'autorité, pratiquant un discours clair dans une langue parfaite, pour une conférence remarquable de précision. Nous étions subjugués, la psychanalyse était intégrée dans le champ des savoirs relatifs aux soins. Il nous dit aussi un mot sur les travaux d'amélioration entrepris dans l'hôpital. Attentif aux modifications du traitement apportées par la vie institutionnelle Roger Misès aimait rappeler l'anecdote suivante. Un des rituels de la Fondation Vallée était le voyage annuel en Normandie d'une délégation des pensionnaires sur la tombe du fondateur, Hippolyte Tranquille Vallée (1816-1885). Une année, un des enfants sachant lire s'offusqua de ce que l'inscription funéraire portée sur la tombe rappelait l'action bienfaitrice de Vallée pour les enfants idiots, comme on disait au XIX ${ }^{\mathrm{e}}$ siècle. Roger Misès supprima alors le voyage annuel devenu inutile, la remarque de l'enfant traduisant les effets d'une meilleure prise en charge des soins dans l'hôpital. Il y avait toujours quelque chose à apprendre des moments de détente que le Professeur ménageait à ses auditeurs, durant lesquels il s'adonnait parfois à la pratique d'un humour féroce et joyeux. À cette première rencontre d'autres ont succédé, dans des colloques, des congrès. Roger Misès présidait, discutait ou intervenait, toujours la voix haute et claire, le discours précis, l'engagement présent. À chaque rencontre nous échangions quelques mots, il était très sollicité. J'ai par la suite travaillé avec Roger Misès dans le Conseil scientifique de l'Association des ITEP et de leurs réseaux (AIRe). Il apporta son soutien technique, théorique et clinique dans l'élaboration du Décret $n^{\circ} 2005$ - 11 du 6 janvier 2005 fixant les conditions techniques d'organisation et de fonctionnement des Instituts Thérapeutiques, Éducatifs et Pédagogiques, constituant les ITEP en remplacement des Instituts de Rééducation (IR). Il a été plus critique sur la Circulaire interministérielle du 14 mai 2007 relative aux instituts thérapeutiques, éducatifs et pédagogiques et à la prise en charges des enfants accueillis, à cause de l'importance donnée à l'Éducation nationale dans l'organisation du parcours, en opposition aux soins. Et on sait combien on lui est redevable des son engagement contre certaines dispositions automatiques de la Loi n 2005-102 du 11 février 2005 pour l'égalité des droits et des chances, la participation et la citoyenneté des personnes handicapées. Dans une actualité récente, en 2012, il s'est opposé aux recommandations de la Haute Autorité de Santé (HAS) sur les pratiques “ non consensuelles » de la psychanalyse et de la psychothérapie 
institutionnelle auprès d'enfants autistes. Il voulait que la clinique soit “ à visée mutative ", qu'elle permette le changement (au sens psychothérapeutique) plus que le progrès (au sens scolaire), et qu'elle soit multidimensionnelle. Il soutenait aussi l'idée d'une multifactorialité des troubles en dehors de toute condition hégémonique d'une discipline sur une autre, il suffisait d'établir clairement la direction de la cure. C'est un travail à continuer, pour lequel de plus en plus de personnes, d'organismes et d'associations professionnelles se chargent dorénavant.

Nous reproduisons deux textes du Pr Roger Misés : la conférence qu’il a donnée lors des journées d'études de la Fondation John Bost en mars 2011 et son article paru dans La Lettre de Psychiatrie Française, n 205, février 2012. 


\section{We interventions de soin chez les enfants et les adolescents : les effets de l'application de la loi du 11 février 2005[1]}

Roger Misès ${ }^{[2]}$

Avertissement : Le champ du handicap psychique ou mental est en pleine expansion, on y fait entrer des enfants à qui des mesures conjointes incluant le soin permettent parfois l'accès à une réintégration étendue. Naturellement, il est essentiel que les mêmes dispositifs d'orientation et d'intervention soient employés pour des cas très favorables et pour d'autres qui se révèlent peu accessibles à des mesures curatives : les psychiatres connaissent bien les dangers d'un clivage entre " aigus » et " chroniques », entre malades et handicapés; le soin doit être ouvert à tous, à travers des approches multidimensionnelles où il s'articule avec des mesures de compensation. Mais, on le sait, les parents, appelés aujourd'hui à formuler un " projet de vie ", font rarement une demande spontanée de soin, si ce n'est pour des actions à visée symptomatique. De surcrồt, à la MDPH les procédures ne répondent guère aux exigences requises pour l'ouverture d'un débat sur les actions de soin qui seraient les mieux adaptées à la singularité de chaque cas.

Le souci exprimé ici de maintenir la possibilité d'un accès aux soins, sous leurs aspects divers, n'est nullement en contradiction avec la reconnaissance des avancées qu'autorise la loi du 11 fésrier 2005.

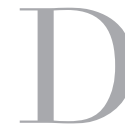

e nombreux praticiens de terrain se demandent aujourd'hui si, chez les enfants et les adolescents, des interventions de soins à visée mutative sont encore réalisables après les révisions qui ont été introduites par la loi du 11 février 2005. Pour éclairer le débat, j’avancerai quelques repères.

I. Dans les années 1950/1960, des orientations curatives se sont dessinées en prenant appui sur des critères dynamiques, structuraux, évolutifs qui ont permis de porter un regard nouveau sur des pathologies précoces et graves où les handicaps sont souvent au premier plan.

[1] Au mois de mars 2011, l'association John Bost tenait ses journées d'étude dans son établissement de La Force (Dordogne). C'est la conférence du Pr Roger Misés, donnée à cette occasion, que nous reproduisons ici. L'avertissement est de sa main. (NDLR).

[2] Professeur émérite de pédopsychiatrie, Université Paris Sud. 
Ces enfants se sont montrés accessibles à des interventions multidimensionnelles qui permettent de soutenir l'accès à une réintégration étendue : assurément, les résultats varient d'un sujet à l'autre en fonction de multiples facteurs, mais d'indéniables transformations ont été relevées chez beaucoup de ces sujets au plan psychopathologique, conjointement avec des progrès observés dans l'intégration familiale, scolaire, sociale.

Parallèlement, les explications d'ordre étiologico-clinique où s'opposaient organogenèse et psychogenèse ont cédé la place à des approches intégratives tandis que l'accent était mis sur l'approfondissement des critères psychopathologiques. Sur ces bases ont pu se développer des approches multidimensionnelles articulant étroitement le soin, sous ses diverses formes, avec l'éducation et la pédagogie - ceci dans le cadre de pratiques en réseau où la priorité a été donnée au maintien de l'enfant dans son milieu habituel - sa famille, son école.

Sous ces orientations, les interventions de soin et les mesures de compensation des handicaps se soutiennent mutuellement et elles entrent dans des interactions dialectiques d'une particulière complexité ce qui exclut radicalement les clivages établis entre inné et acquis, entre maladie et handicap, entre soin et éducation.

II. Les actions menées auprès des enfants et des adolescents se sont appuyées sur ces bases, avec le plein appui des pouvoirs publics, jusque dans les années 1980. À ce moment, de nouvelles orientations venues des États-Unis ont introduit les critères et les concepts du DSM-III ou de l'Evidence Based Medecine : ici l'évaluation se fonde sur le seul recueil des données immédiatement objectivables et les anomalies constatées se trouvent expliquées par des dysfonctionnements d'ordre neurologique exclusif d'où découle la notion d'une “ irréversibilité » des troubles. Cette irréversibilité fait écarter, par principe, la visée mutative, cependant des mesures thérapeutiques, rééducatives ou de soin, restent à la disposition : elles se trouvent surtout utilisées sous une visée normative, centrée sur des aspects partiels, au point de se trouver rangées parfois parmi les modes de compensation des handicaps.

Il me semble important de noter que, contrairement à ce qui s'était produit aux ÉtatsUnis, ces conceptions réductrices n’ont pas suscité en France une adhésion massive.

- Certaines Associations de parents opposés aux orientations dynamiques y ont apporté un vif soutien, mais d'autres Associations ont maintenu les liens établis antérieurement avec des professionnels engagés dans des approches ouvertes.

- Du côté des équipes, des réserves se sont exprimées, dans la plupart des cas, envers le modèle proposé ; par contre un intérêt accru s'est manifesté pour les recherches menées sur le terrain de sciences cognitives, des neurosciences, des sciences de l'éducation. En a découlé une diversification des supports conceptuels et des pratiques, mais sans que soient remis fondamentalement en question l'articulation entre les actions de soin et les mesures qui visent à la compensation des handicaps. Dans ces conditions, les liens tissés antérieurement entre les 
membres des équipes de psychiatrie et les enseignants se sont maintenus dans la reconnaissance mutuelle de la place tenue par chacune des parties.

- Du côté des pouvoirs publics, un évident intérêt s'est manifesté pour les conceptions venues des USA, dans la mesure où elles s'accordaient avec les préoccupations ministérielles relatives à une réduction des dépenses de santé et au renforcement des contrôles gestionnaires, néanmoins, les textes législatifs et réglementaires ont, pour l'essentiel, continué à soutenir les principes préexistants. Quelques exemples : la loi de 1975 sur les personnes handicapées a permis, jusqu'en 2005, d'assurer une représentation paritaire entre les équipes psychiatriques et les enseignants, ce qui a appuyé le développement des actions conjointes ; dans le même esprit en 1989 la révision des annexes 24 a mis l'accent sur l'élaboration, pour chaque enfant, d'un projet individualisé où le soin trouve toute la place qu'il requiert ; la circulaire du 2 décembre 1992 relative à la sectorisation en psychiatrie infanto juvénile a proposé de son côté, pour “ l'autisme et les autres formes de psychose des enfants et des adolescents ", que les équipes puissent “ assurer le diagnostic le plus précoce possible » et qu'elles mettent en œuvre " des thérapies appropriées ainsi que les mesures d'accompagnement nécessaires sur le plan éducatif et pédagogique "; enfin, la loi du 2 février 2002 a soutenu aussi le décloisonnement des dispositifs, avec le souci affirmé d'améliorer les réponses faites aux personnes qui relèvent simultanément des soins et d'un accompagnement social.

Avec les appuis trouvés ainsi sur différents plans, ont pu se développer des actions conjointes qui ont été soutenues à la fois par les praticiens et par des Associations de parents. En témoigne la parution en 1997 d'un ouvrage collectif que j’ai dirigé avec Ph. Grand ${ }^{[3]}$, représentant d'une Association de parents : plusieurs collègues présents aujourd'hui (C. Aussilloux ; J. Constant ; Ph. Gabbai ; J. Hochmann) y ont apporté d'importantes contributions ; on constate dans ce livre que les progrès réalisés concernent tous les enfants et adolescents handicapés repérables dans le champ de la Santé Mentale. Les sujets à autonomie très réduite sont abordés par des mesures de soin spécifiques et non spécifiques, associées à des interventions qui visent à la socialisation et à la déségrégation ; en même temps une attention particulière est portée au maintien des liens avec la famille. Pour d'autres enfants, accessibles à des approches multi disciplinaires, les moyens éducatifs développés sous des aspects systématisés trouvent leur place dans des interventions conjointes qui peuvent inclure une visée thérapeutique affirmée. Enfin, du côté des dysharmonies psychotiques qui disposent déjà de notables capacités adaptatives, le soin dans sa visée mutative prend une place centrale parmi les actions coordonnées qu'on met en œuvre dans un espace élargi. Ainsi le souci d'ajuster les mesures de soin et d'accompagnement aux particularités de chaque enfant par des contributions issues de différents courants s'est manifesté 
ici de façon que le débat d'idées et la recherche de solutions pragmatiques puissent se substituer aux affrontements passionnels.

III. C'est dans ce contexte, en somme très ouvert, qu'a été discuté, un peu plus tard, le projet relatif à la loi du 11 février 2005 : ce projet a été soutenu par la grande majorité des praticiens, dans la mesure où il offrait des avancées notables sur les terrains de la compensation, de l'adaptation, de la réhabilitation, il laissait également présager une plus grande efficacité dans la lutte contre les discriminations. Certes, des critiques ont été formulées, en particulier pour l'usage du terme " handicapé psychique » : or, selon moi, cette appellation qui répond au vœu des parents, se justifie car la nouvelle législation est venue en remplacement de la loi de 1975 sur « les personnes handicapées ", et, de plus, la loi de 2005 fait mention, de façon explicite, d'un recours possible au soin ou à la thérapeutique, y compris à travers l'appel aux équipes et aux dispositifs du champ sanitaire ou médico social.

Plus importantes, m’apparaissaient, à ce moment, les questions en suspens, relatives à la place qui serait donnée aux équipes psychiatriques dans les nouvelles procédures, avec le souci d'assurer le maintien des actions conjointes, notamment la coopération avec les enseignants ; à ce propos, si l'on se référait aux précédents textes législatifs ou réglementaires, on était en droit d'espérer que les équilibres établis seraient maintenus. On le sait, ces espérances ont été déçues ; j'évoquerai seulement quelques faits parmi les plus démonstratifs.

- En 2008, à l'occasion de la présentation du Plan relatif aux troubles envahissants du développement (TED), la Secrétaire d'État à la Solidarité a exposé que « de nouvelles mesures éloignées de la psychanalyse et de la psychiatrie seront désormais appliquées [...] de façon à offrir des structures plus clairement éducatives ${ }^{\left[{ }^{[4}\right.}$. Ces dispositions ne concernent plus, comme par le passé, les cas très rares qu'on classait sous la rubrique de l'autisme ou des psychoses précoces : aujourd'hui avec les concepts et les critères du DSM, repris par la CIM-10, le nombre des enfants classés TED a subi un accroissement prodigieux, en 15 ans le taux de prévalence est passé de 4 à 5 pour 10000 à 1 pour cent. Ceci répond à l'inclusion dans ce cadre de faits cliniques rangés antérieurement sous d'autres rubriques. Avec des critères psychopathologiques discriminants, on constate que parmi les enfants classés désormais TED, certains, comme les dysharmonies psychotiques, occupent une place importante et sont pleinement accessibles à des interventions multidimensionnelles menées sous une visée curative.

Les directives ministérielles remettent donc en question des pratiques de soin dont les effets positifs avaient été démontrés : si l'on excepte, en effet, les tenants d'une irréductibilité absolue des troubles, un accord s'était établi entre la plupart des cliniciens pour considérer que certaines pathologies du jeune âge pouvaient bénéficier d'approches multidimensionnelles à visée réintégrative, sous trois conditions : que l'on intervienne tôt, avec des moyens adaptés et

[4] Le Monde, 4 avril 2008. 
en coopération étroite avec les parents. Sous les nouvelles directives ministérielles, on garde le principe d'une intervention précoce, on donne une place élargie aux parents, mais on écarte la visée mutative.

- Sous des formes moins évidentes, d'autres révisions sont intervenues parallèlement, en particulier à travers les textes d'application de la loi du 11 février 2005 : ici aussi, le Ministère de la Santé à dessaisi les psychiatres et leurs équipes de beaucoup de leurs fonctions. On constate, par exemple, que les textes d'application, dans leur très grande majorité, émanent seulement de l'Éducation Nationale et qu'ils confèrent une place dominante, voire exclusive, aux perspectives pédagogiques dans les procédures d'orientation et de suivi des enfants scolarisés.

- Cette prééminence est revendiquée hautement par l'Éducation Nationale, ainsi lors d'un colloque réunissant psychiatres et enseignants ${ }^{\left[{ }^{5}\right.}$, un inspecteur d'académie considère que, sous les nouvelles orientations, “ l'enfant handicapé est devenu un élève handicapé... par la seule vertu de l'application de la loi [...] la notion d'intégration se trouve également invalidée, la capacité même à être scolarisé n'est plus d'une importance première »; sous ces réorientations, le projet personnalisé de scolarisation devient prépondérant sur tout autre repère et l'enseignant peut s'affirmer comme le "garant exclusif de l'évolution de l'enfant ", à travers un processus où le soin ne peut plus apparaître que sous la forme de prestations d'accompagnement.

- Sur ces bases, toutes les études et les enquêtes en cours font voir que les équilibres antérieurement établis entre les équipes pédopsychiatriques et les équipes pédagogiques se trouvent profondément remis en question, mais, plus encore, certains textes réglementaires ont été modifiés afin d'assurer la primauté du pédagogique. On le constate avec les instituts thérapeutiques, éducatifs et pédagogiques (ITEP) où la dimension du soin trouvait toute sa place avec le décret fondateur du 6 janvier 2005 qui soutenait la primauté des actions coordonnées, menées, de façon paritaire, entre tous les intervenants ; or, par application de la loi du 11 février 2005, une circulaire du 14 mai 2007 a introduit comme référence dominante " la pédagogie des élèves handicapés ». Cette innovation bouleverse les rapports paritaires établis précédemment entre le soin, l'éducation, la pédagogie ; certes, dans certains passages de la récente circulaire, les sujets reçus en ITEP sont encore reconnus comme des pathologies de la personnalité de type limite et dominées par le recours à l'agir, mais désormais sous les orientations dominantes, ces enfants apparaissent prioritairement comme des "élèves handicapés " et ils sont soumis aux décisions de la MDPH qui les envisage sous l'angle d'une perte d'autonomie justifiant l'insertion dans " le programme départemental d'accompagnement du handicap », en vue d'une " compensation des défauts d'apprentissage scolaire ": il n'est donc plus concevable d'établir un projet personnalisé, qui, en prenant en compte le risque d'évolution vers la psychopathie donnerait aux soins la place qu'on leur reconnaissait antérieurement. De plus, à la MDPH, avec l'établissement du projet de scolarisation, la priorité est accordée à la désignation d'un référent scolaire, personnage clé qui intervient ensuite à l'intérieur de l'ITEP pour s'assurer de l'exécution conforme du projet scolaire préétabli. Sous ces directives, on méconnaît parfois,

[5] Cf. Handicap mental de l'enfant. Scolarisation, intégration, égalité des chances? Psychiatrie Française, vol. XXXIX, 02/08, Novembre 2008. 
gravement, l'influence que des composantes d'ordre psychopathologique peuvent exercer sur les difficultés scolaires - qu'on met pourtant au centre de l'évaluation et des pratiques. Ceci est d'autant plus regrettable qu'antérieurement, la plupart de ces enfants répondaient de façon favorable à des mesures conjointes.

Il faut rappeler que, sous les approches multi dimensionnelles menées dans des dispositifs en réseau, une place entière était faite aux enseignants dans un projet aux multiples facettes. Ainsi, dans sa classe, un professeur se centrait assurément sur les difficultés d'adaptation de l'élève et sur la réalisation des apprentissages scolaires, mais, dans le même temps, à travers les liens tissés entre les intervenants, ce professeur en arrivait également à tenir une place importante dans un projet collectif qui comportait une dimension de soin - et cela sans qu'il y ait confusion entre ses fonctions propres et celles qui incombent aux éducateurs et aux thérapeutes.

Les temps semblent donc révolus où, en se décalant ainsi, quelque peu, de sa fonction principale, un enseignant pouvait tenir ces positions ouvertes : aujourd'hui, elles lui sont interdites par les procédures instaurées, mais, peut-être plus radicalement encore, par le projet hégémonique que développe l'Éducation Nationale à l'égard des enfants scolarisés où la visée pédagogique se veut exclusive de toute autre.

Les obstacles que rencontrent les équipes de Santé Mentale ne dépendent pas, je l'ai déjà dit, de la Loi elle-même, mais de ses textes d'application. D'autres influences corrélatives s'exercent dans ce contexte : entre autres, le retrait du soutien apporté aux équipes psychiatriques par les pouvoirs publics, la référence de plus en plus fréquente à l'irréductibilité des troubles et, à partir de là, l'exclusivité donnée aux mesures de compensation des handicaps - sans oublier la pénurie extrême en personnel soignant dans le médico social.

Lors d'une journée d'études de la Société française de psychiatrie de l'enfant et de l'adolescent et des professions associées, A. Crochette ${ }^{[6]}$ en fait le constat : “ Les procédures d'orientation en lien avec la mise en place des MDPH ont profondément modifié le cadre, les contenus, les enjeux des échanges entre l'école et la pédopsychiatrie, bouleversant les formes, bonnes ou critiquables, du parler ensemble autour des besoins d'orientation et de soins de l'enfant, défaisant les liens tissés en trente années de mise en application de la loi de 1975 » désormais, l'enfant apparaît “ dispersé, éclaté entre de multiples prises en charge, habituellement cloisonnées ».

Cette révision en cours des concepts et des pratiques, évoque par son étendue " le grand renversement "survenu aux USA dans les années 1980 ; l'hégémonie affirmée par le courant dominant impose, en effet, des concepts et des pratiques qui font écarter la possibilité d'un recours aux interventions de soin qu'on utilisait antérieurement avec des résultats favorables. Ceci justifie une interrogation sérieuse sur les 
moyens à mettre en œuvre pour, à nouveau répondre, aux exigences d'ordre clinique et éthique ${ }^{[]]}$qui permettent de prendre en considération les potentialités évolutives de chaque enfant en lien avec ses capacités adaptatives.

Si les pouvoirs publics souhaitent, un jour, intervenir en ce sens, ils pourront prendre appui sur des principes que valorise, à juste titre, la loi du 11 février 2005 : l'accessibilité, la solidarité, la participation. Il importe, en effet, d'assurer l'accessibilité aux soins des enfants et des adolescents scolarisés; d'aider au rétablissement d'une véritable solidarité entre les intervenants, de permettre une authentique participation aux membres des équipes de santé mentale. Ceci me paraît réalisable par des mesures d'ordre purement réglementaire et en référence à la Loi du 11 février 2005, mais aussi à celle du 2 février 2002 dont l'objectif est d'améliorer les réponses données aux personnes qui relèvent, à la fois, du soin et d'un accompagnement social ». 


\section{We Ouvrir l'espace pour un échange ouvert ${ }^{[8]}$}

Roger Misès ${ }^{[9]}$

utour des affrontements sur l'autisme, je pense qu'il est important de s'accorder sur des principes clairs et accessibles à tous, susceptibles de permettre le dépassement des controverses stériles et en veillant à garantir un respect suffisant pour l'opinion des personnes qui ne partagent pas les mêmes options. En 1990, j'avais soutenu à la Commission des maladies mentales les orientations de la circulaire parue plus tard le 11 décembre 1992, sur la sectorisation en psychiatrie infantile, ceci vingt ans après le texte fondateur du 11 mars 1972. S'y trouvent évoqués les problèmes particuliers posés dans l'abord de “certains enfants souffrant d'autisme ou d'autres formes de psychoses infantile » - rangés aujourd'hui parmi les troubles envahissants du développement (TED) ou les troubles du spectre autistique (TSA) avec un accroissement fantastique de leur nombre.

Je cite intégralement le texte : “Il est rappelé que les secteurs de psychiatrie infanto-juvénile ont vocation à prendre en charge ces enfants et adolescents : il s'agit de permettre un diagnostic le plus précoce possible - tel qu'il s'est développé ces dernières années, notamment du fait de la collaboration avec les pédiatres -, de mettre en ouvre précocement des thérapeutiques appropriées, ainsi que les mesures d'accompagnement nécessaires sur le plan éducatif et pédagogique, et de préparer à l’adolescence le suivi vers des structures diversifiées. À toutes ces étapes, une attention particulière devra être portée à la participation des familles. Ceci garantit contre une dérive inquiétante qui dessaisirait les équipes psychiatriques de leurs actions auprès des autistes. ”

En raison des revirements majeurs de la politique de santé sous les pressions de certains groupes de parents, ces perspectives n’ont pas été appliquées et cela sans que puisse s'ouvrir un véritable débat.

Cependant, en France, des recherches et des études ont été menées sous différents angles ; elles ont conduit à intégrer les contributions nouvelles issues des neurosciences, des sciences cognitives, des sciences de l'éducation, de la sociologie, tout en faisant une place aux données d'ordre psychopathologique provenant, entre autres,

[8] R. Misès, La Lettre de Psychiatrie Française, n²05, février 2012.

[9] Professeur émérite de pédopsychiatrie à l'Université Paris Sud. Membre d'honneur de l'Association Française de Psychiatrie. 
des éclairages portés par la psychanalyse. Ceci permet aux intervenants d'apprécier la singularité de chaque cas et de rechercher, en lien étroit avec les parents, les éducateurs, les enseignants, une solution adaptée que l'on fait évoluer à mesure, et ceci à la fois dans la dimension du handicap et d'un point de vue psychopathologique, car certains sujets relèvent assurément de mesures préventives et curatives. Une étude longitudinale menée à la Fondation Vallée, avec l'appui de l'Inserm, auprès d'un groupe d'enfants gravement handicapés suivis jusqu'à l'âge adulte, a montré les effets positifs de ces approches multidirectionnelles, à la fois sur le plan du handicap et sur celui des remaniements structuraux.

C'est dire que la majorité des équipes françaises de psychiatrie infanto-juvénile ont soutenu les avancées proposées par la loi du 11 février 2005 sur le terrain de l'accompagnement, de l'intégration, de la réhabilitation, mais ces mesures ne sauraient être, par principe, et par décision parlementaire, coupées des approches qui permettent d'intervenir conjointement auprès de certains sujets reconnus sur des critères différentiels et évolutifs.

Aujourd'hui, l'essentiel est donc bien d'ouvrir l'espace et de se donner le temps de la réflexion, pour un échange ouvert, hors de toute décision précipitée qui mènerait à l'irréparable ». 